\title{
«Rosenstrumpf und dornencknie»
}

Maike Rotzoll

Korrespondenz:

Dr. med. Maike Rotzoll Institut für Geschichte und Ethik der Medizin Ruprecht-Karls-Universität Heidelberg

Im Neuenheimer Feld 327 D-69120 Heidelberg

maike.rotzoll@histmed. uni-heidelberg.de
Das Medizinhistorische Museum der Universität Zürich zeigt in einer Sonderausstellung Werke von Patientinnen und Patienten der Psychiatrischen Pflegeanstalt Rheinau aus den Jahren 1867 bis 1930. Aus der faszinierenden Sammlung werden etwa Werke von Lisette H. ausgestellt, die mit Matratzenfüllmaterial strickte, oder von Jeanne Natalie Wintsch, die verschlüsselte Botschaften stickte. Als Einführung in die Sonderschau bespricht die Medizinhistorikerin und Psychiaterin Maike Rotzoll von der Universität Heidelberg hier den gleichzeitig erschienenen Ausstellungsband.

(Red.)

«Reif für die Insel» - mit dieser Formulierung kann man in heutigen Alltagsgesprächen konfrontiert werden. Dann ist etwas wie «erholungsbedürftig» gemeint. Doch stecken tiefere Bedeutungsschichten hinter dieser Wendung, neben dem Abstand vom Alltag, der sicherlich impliziert wird? Grosse Inseln sind wie ferne Kontinente. Dorthin kann man sehnsuchtsvoll blicken oder tatsächlich auswandern aber das ist wohl nicht gemeint. Kleine Inseln haben vielfältige Funktionen: Idyllische Orte des Rückzugs und der Weltabgewandtheit können sie sein, mit Klöstern oder Schlössern, wie die Reichenau im Bodensee oder die Isola Bella im Lago Maggiore. Als
Nach einem Besuch der bei Wien gelegenen Heilund Pflegeanstalt «Am Steinhof» im Jahr 1919 wählte der Journalist Josef Roth (1894-1939) zu ihrer Beschreibung eben diesen Begriff: «Insel der Unseeligen». Eine Insellage im übertragenen Sinne könnte man vielen der im 19. und 20. Jahrhundert eingerichteten «Irrenanstalten» bescheinigen, als fernab gelegene Orte der Ausgrenzung. Einrichtungen für psychisch Kranke, die tatsächlich auf einer Insel lagen, wie vor Venedig San Servolo oder die Rheinau im Kanton Zürich, waren also Inseln in doppeltem Sinne.

Wen der Fährmann übersetzte, über den Strom zur Insel, über den Rhein zur Rheinau, der musste nach 1867 damit rechnen, dass die Verbannung unumkehrbar sein könnte. Denn das im Barockstil erbaute ehemalige Kloster in reizvoller Lage wurde als Pflegeanstalt für «Unheilbare» genutzt. Sie kamen aus der psychiatrischen Klinik Burghölzli oder von anderswo im Kanton Zürich, jedenfalls hatte man alle Hoffnung auf ihre Heilung aufgegeben. Streng nach Geschlecht getrennt, lebten dort anfangs um 400, später - nach der Erweiterung der Anstalt um den Bereich Neu-Rheinau auf dem Festland bis zu 1200 Insassen gleichzeitig. Seit der Schliessung der Inselklinik im Jahr 2000 wird lediglich das Areal

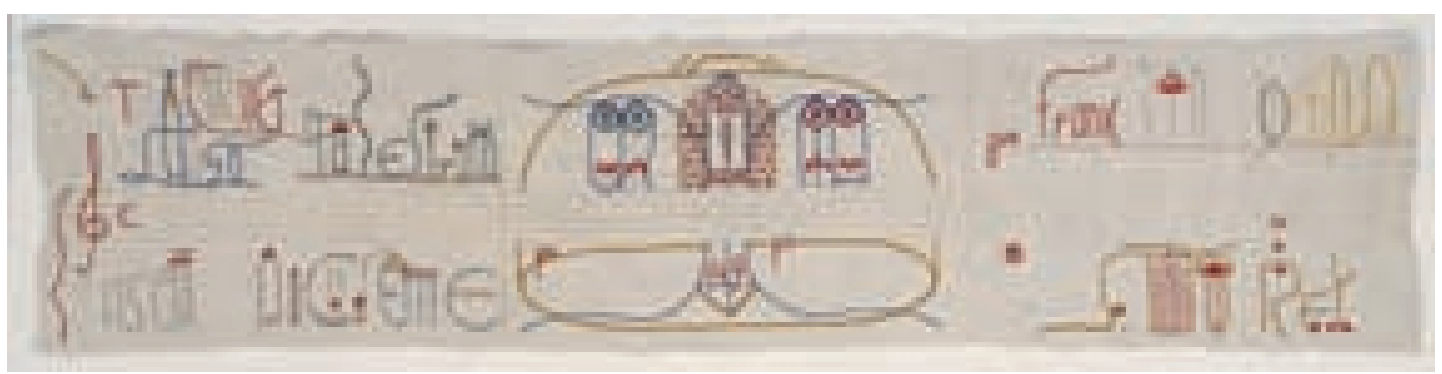

Jeanne Natalie Wintsch (1871-1944): «L’adoré», Stickerei (um 1924). @ Kanton Zürich, Baudirektion, Immobilienamt, Slg. Rheinau.

letzte Ruhestätte dient San Michele in der venezianischen Lagune, die Friedhofsinsel. Nicht weit davon geben die Inseln Lazzaretto Vecchio und Lazzaretto Nuovo durch ihre Namen Kunde von ihrem früheren Zweck: durch Krankheit aus der Gesellschaft Ausgestossene aufzunehmen, im Fall einer Seuche mehr die (noch) gesunde Bevölkerung als die Kranken zu schützen - auch in diesem Fall war der Abstand zum Festland das entscheidende Kriterium für die Wahl des Ortes. Auf einsamen Inseln kann man stranden oder auf sie verbannt werden. Weniger einsam geht es $\mathrm{zu}$, wenn auf Inseln Gefängnisse oder «Irrenanstalten» eingerichtet werden. Es gibt Inseln der Seligen, möchte man hoffen, und Inseln der Unseligen, so scheint es. auf der Landseite weiter als psychiatrische Klinik genutzt.

Zurück blieb ein Kunst-Schatz, der Anlass zu dem hier zu besprechenden Katalog gab: Nicht von ungefähr geht von diesem Buch die Aura eines Schatzkästchens aus. Den Deckel von «Rosenstrumpf und dornencknie» ziert kostbar und filigran wirkendes, mit dunkler, lateinischer Schrift geschmücktes Besteck, doch ist es aus schlichtem Holz geschnitzt. Innen wird ein Teil des gehobenen Kunstschatzes vorgestellt: fein ziselierte Federzeichnungen subtiler Erfindungen, kunstvolle Seegras-Strickereien, schablonenartig stilisierte Chroniken des Insellebens und flimmernde Ornamente, gekonnte Darstellungen des stets gleichbleibenden Landschaftsmotivs «Rhein bei 
Rheinau», bedeutungsgeladen-rätselhafte Stickereien, zart, in paradiesischen Farben gehalten, anmutigspielerische Gruppen von Lindenholzhasen. Vor dem inneren Auge des Betrachters mutiert die Barockkloster-Anstalt zum Zauberschloss mit zahlreichen Erkern und Türmchen, und in jede der verwunschenen Ecken hat sich ein Künstler oder eine Künstlerin mit seinem oder ihrem Werk wie in einen Kokon eingesponnen.

Doch dies kann nur ein kleiner Teil der Wahrheit gewesen sein, wenn auch in der Pflegeanstalt Rheinau künstlerische Tätigkeit angesehener war als Nichtstun. Denn der Anstaltsalltag mit seiner Monotonie, seinem starren Ordnungssystem, den Entbehrungen und der omnipräsenten Arbeit muss die Realität der Patienten/Künstler deutlich stärker bestimmt haben. Auch dies war die Pflegeanstalt Rheinau: ein streng verwalteter, gigantischer Gutshof, ein Arbeitshaus ohne Lohn, ein Aufbewahrungsort für nicht Arbeitsfähige mit einem hierarchisch abgestuften System von Mikrokosmen, an dessen Ende die Stationen für «Unruhige» standen.

Die Herausgeberinnen des Buches «Rosenstrumpf und dornencknie. Werke aus der Psychiatrischen Pflegeanstalt Rheinau 1867-1930», die Kunsthistorikerinnen Katrin Luchsinger (Leiterin des Forschungsprojektes «Bewahren besonderer Kulturgüter») und Monika Jagfeld (Leiterin des Museums Lagerhaus in St. Gallen), die Historikerin Iris Blum sowie die Kunstlehrerin Jacqueline Fahrni stellen eine Auswahl der

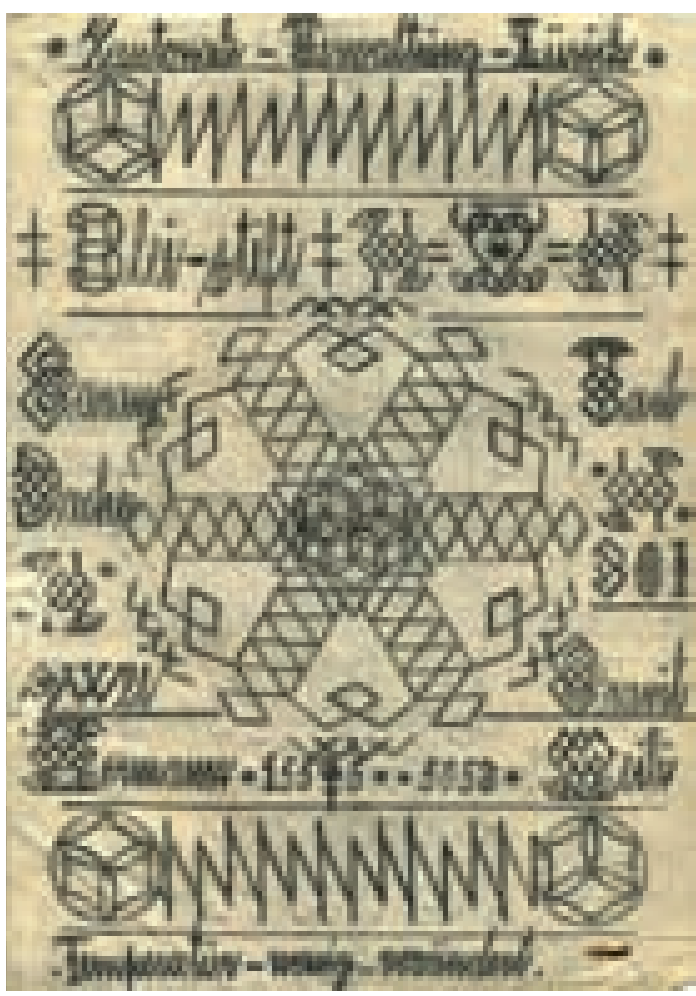

Hermann M. (1894-1943): «Temperatur-wenig-verändert», undatiert. (c) Kanton Zürich, Baudirektion, Immobilienamt, Slg. Rheinau.
«Sammlung Rheinau» der Öffentlichkeit vor. Der mit Bildtafeln, Schwarz-Weiss-Abbildungen und Fotografien reich ausgestattete Band begleitete und begleitet die gleichnamigen Ausstellungen im Museum Lagerhaus St. Gallen (Dezember 2010 bis März 2011), ab Juni 2011 im Medizinhistorischen Museum Zürich und im Jahr 2013 im Kloster Rheinau. Doch ist er weit mehr als ein Katalog.

\section{Mehr als ein Katalog}

Nach Gruss- und Geleitworten beginnt das Buch mit Luchsingers souveräner Einführung in das Ausstellungsprojekt, die Geschichte der Anstalt und der Sammlung bis zur Schliessung der Inselklinik. Die mit ihren 825 Werken im Vergleich zur Heidelberger Sammlung Prinzhorn kleine, in sich geschlossene Kollektion ist laut Luchsinger ein «historisches Dokument und eine Kunstsammlung von ausserordentlicher Poesie und Komplexität». Eindrucksvoll stellt Blum in ihrem Beitrag das Praxisfeld Arbeit in der Rheinau vor. Was wurde nicht alles in der «Staatsdomäne» für die Anstaltsküche und an Vieh gehalten, und dennoch blieben die Insassen häufig hungrig. Wenn auch die Aufzählung der Produkte an ein «Schlaraffenland» denken lässt, war die Anstalt doch eher ein «Arbeitsland», so das Fazit von Blum. $\mathrm{Zu}$ Recht weist sie darauf hin, dass längst vor dem Siegeszug der sogenannten «aktiveren Therapie», vom deutschen Anstaltspsychiater Hermann Simon (1867-1947) in den 1920er Jahren propagiert, Arbeit ein fester Bestandteil des Anstaltslebens war. Man billigte ihr eine therapeutische Funktion zu - deswegen mussten die Patientinnen und Patienten auch keinen Lohn erhalten - und versuchte, einen grösstmöglichen Prozentsatz der Insassen zu beschäftigen, das verbilligte auch die Versorgung. Neu an Simons Konzept war später lediglich der allumfassende Ansatz mit der Ausdehnung auf akut Kranke.

Die zehn prägnanten Kurzbiografien stammen von Fahrni und Luchsinger. Mit einer Ausnahme werden die Nachnamen der Künstlerinnen und Künstler anonymisiert und somit ihr Patientenstatus akzentuiert.

Dann stellt Luchsinger ausführlicher Erfindungen des ehemaligen Eisenbahnangestellten Heinrich B. (1863-1926) vor. Sie dokumentieren hohe technische Präzision und Ästhetik, wenn sie auch nicht alle praktikabel gewesen sein mögen. Auch das CEuvre von Hermann M. (1894-1943) beschreibt Luchsinger. Über die Jahre seines Schaffens von 1927 bis 1933 nahmen seine Dokumentationen des Anstaltsalltags, seine Dichtungen und ornamentalen Zeichnungen einen immer abstrakteren, verschlüsselten Charakter an, bis sie zur Geheimschrift wurden. Zurück bleiben bestechende Lautakrobatik und Zeichen, die die besondere Qualität besitzen, den Betrachter in den Zustand der Trance zu versetzen.

Bettina Brand-Claussen, frühere Kuratorin der Heidelberger Sammlung Prinzhorn, widmet sich der 


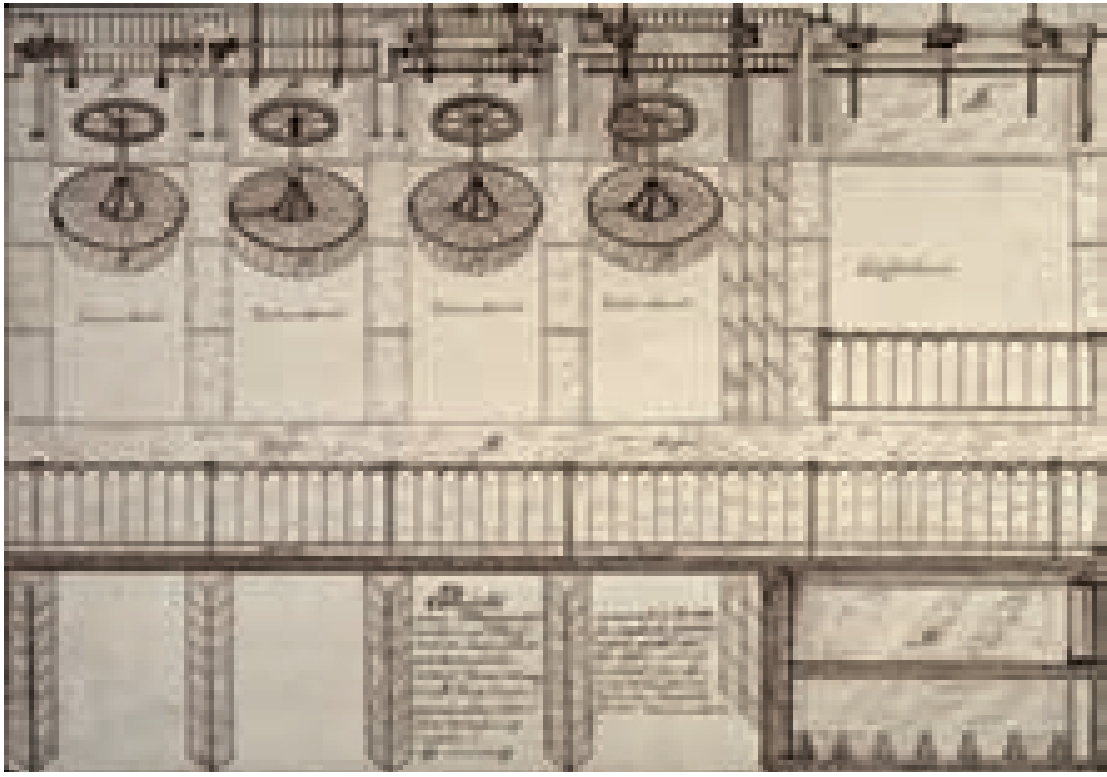

Heinrich B. (1863-1926): «Projekt eines Rheinwasserwerkes mit Schiffschleuse» (1916). (c) Kanton Zürich, Baudirektion, Immobilienamt, Slg. Rheinau.

einzigen Frau, deren Werk in diesem Katalog ausführlicher präsentiert wird. Dies ist sicher kein Zufall, hatte die Autorin doch 2004 in Heidelberg die Ausstellung «Irre ist weiblich» kuratiert (gemeinsam mit Viola Michely). Im gleichnamigen «Frauenkatalog» spielte die im Burghölzli und in der Rheinau psychiatrisierte Klavierlehrerin und Stickerin Jeanne Natalie Wintsch (1871-1944) eine grössere Rolle, deren Werke teils in Heidelberg, teils in der Rheinau erhalten sind. Die phantastischen Arbeiten voller RätselBotschaften setzen auch in dem aktuellen Band einen Glanzpunkt. In dieser fast märchenhaften Geschichte siegte die Phantasie: Es gelang Wintsch, den für sie zuständigen Assistenzarzt für sich einzunehmen und «bestickend» zu überzeugen, so dass sie 1925 nach Hause zurückkehren konnte.

Die Werkvorstellungen schliesst Jagfeld mit Anmerkungen zu Jakob Friedrich W. (1893-1982), der seit 1920 insgesamt 62 Jahre in der Rheinau lebte. 350 Werke, darunter auch zahlreiche Skizzenhefte, haben sich erhalten. Der frühere Versicherungsagent verstand sich als Landschaftsmaler mit dem Ziel, die Natur realistisch wiederzugeben, auch wenn er situationsbedingt sein Sujet kaum variieren konnte.

Die letzen drei Beiträge stellen die Kunstwerke aus der Sammlung Rheinau in einen grösseren Zusammenhang. Auf der Suche nach Möglichkeiten der Annäherung an Werke aus psychiatrischen Anstalten blickt Jagfeld zunächst auf die Rezeptionsgeschichte der Sammlung Prinzhorn zurück: Von der Pathologisierung der Werke aufgrund der psychiatrisierten Autoren bis zu ihrer Anerkennung als Kunst und Beitrag zu einer umfassenden Kulturgeschichte. Daniel Baumann widmet sich in Form eines Exkurses dem letzten Werk des Waldau-Patienten Adolf Wölfli (1864-1930), dem «Trauermarsch» mit seinem klanghaft-kompositorischen Einsatz von Sprache. Dem Thema der Sprache in psychiatrischen Anstalten widmet sich abschliessend der Medizinhistoriker Vincent Barras: Zwischen den Polen Pathologie und Poesie erschliesst sich ein weites Feld, weitgehend neu zu entdecken.

Eine solche Entdeckung sind zweifellos die Gedichte von Hermann M. Jenseits ihrer lautmalerischen Qualitäten verdichten sich manche seiner Wortbilder zu Zeichen für das Leben in der Pflegeanstalt Rheinau (und in anderen psychiatrischen Anstalten seiner Zeit) zwischen Zauberschloss und Arbeitshaus - zwischen «Rosenstrumpf und dornencknie».

\section{Die Ausstellung}

Rosenstrumpf und dornencknie. Werke aus der Psychiatrischen Pflegeanstalt Rheinau 1867-1930. Sonderausstellung bis 12. Januar 2012 im Medizinhistorischen Museum der Universität Zürich. Dienstag bis Freitag 13.00-18.00 Uhr, Samstag/Sonntag 11.00-17.00 Uhr. Öffentliche Führungen und Begleitprogramm unter www.mhiz.uzh.ch

\section{Der Katalog}

Rosenstrumpf und dornencknie. Werke aus der Psychiatrischen Pflegeanstalt Rheinau 1867-1930. Herausgegeben von Katrin Luchsinger, Iris Blum, Jacqueline Fahrni, Monika Jagfeld. Zürich: Chronos 2010; 108 S. 63 Abb. 30 CHF. ISBN 978-3-0340-1057-3. 